\title{
S-Adenosylmethionine, a Promising Antitumor Agent in Oral and Laryngeal Cancer
}

\author{
Laura Mosca, Francesca Vitiello, Martina Pagano, Alessandra Coppola, Roberta Veglia Tranchese, Roberta Grillo, \\ Giovanna Cacciapuoti * and Marina Porcelli * (D)
}

Citation: Mosca, L.; Vitiello, F.; Pagano, M.; Coppola, A.; Veglia Tranchese, R.; Grillo, R.; Cacciapuoti, G.; Porcelli, M. S-Adenosylmethionine, a Promising Antitumor Agent in Oral and Laryngeal Cancer. Appl. Sci. 2022, 12, 1746. https://doi.org/ 10.3390/app12031746

Academic Editor: Maria Contaldo

Received: 26 November 2021

Accepted: 4 February 2022

Published: 8 February 2022

Publisher's Note: MDPI stays neutral with regard to jurisdictional claims in published maps and institutional affiliations.

Copyright: (C) 2022 by the authors. Licensee MDPI, Basel, Switzerland. This article is an open access article distributed under the terms and conditions of the Creative Commons Attribution (CC BY) license (https:// creativecommons.org/licenses/by/ $4.0 /)$.
Department of Precision Medicine, University of Campania “Luigi Vanvitelli”, Via Luigi De Crecchio 7, 80138 Naples, Italy; laura.mosca@unicampania.it (L.M.); francesca.vitiello@unicampania.it (F.V.); martina.pagano@unicampania.it (M.P.); alessandra.coppola@unicampania.it (A.C.); roberta.vegliatranchese@unicampania.it (R.V.T.); roberta.grillo@unicampania.it (R.G.)

* Correspondence: giovanna.cacciapuoti@unicampania.it (G.C.); marina.porcelli@unicampania.it (M.P.); Tel.: +39-081-5667-518 (M.P.); Fax: +39-081-5667-519 (G.C.)

\begin{abstract}
Squamous cell carcinoma of the head and neck (HNSCC), which includes cancers of the oral cavity and larynx, is one of the most common and highly aggressive malignancies worldwide, despite significant efforts committed in recent decades in its detection, prevention, and treatment. The intrinsic or acquired drug resistance during treatment is the main limitation to chemotherapy, increasing mortality and cancer recurrence. Therefore, there is a growing scientific interest in identifying and developing adjuvant chemotherapies able to improve currently available treatments. S-Adenosylmethionine (AdoMet), a safe and nontoxic natural cofactor with pleiotropic effects on multiple cellular processes and the main biological methyl donor in transmethylation reactions, has been considerably studied as a therapeutic compound. Its application, alone or in combination with other drugs, is emerging as a potentially effective strategy for cancer treatment and for chemoprevention. This review summarizes the structural, pharmacological, and clinical aspects of AdoMet and provides an overview of the recent results highlighting its anticancer activity in the treatment of oral and laryngeal cancer, with particular emphasis on its molecular mechanisms and the promising chemoprotective and synergistic effects exerted in combination with cisplatin and specific microRNAs.
\end{abstract}

Keywords: S-adenosylmethionine; oral cancer; laryngeal cancer; microRNA; combination therapy

\section{Introduction}

Oral and laryngeal cancers are a large group of malignancies belonging to head and neck cancers developing from the mucosal epithelium in the oral cavity and larynx [1,2]. Head and neck cancers (HNC) represent one of most frequent cancers worldwide, with squamous cell carcinoma (HNSCC)being the most frequent histologic subtype accounting for about 900,000 cases and over 400,000 deaths annually with poor 5-year overall survival rates [1-3]. Patients suffering from these cancers usually belong to the older age group, and males are about three times more likely to develop it than females [1-3]. The main risk factors associated with oral and laryngeal cancers are tobacco smoking and alcohol consumption. Human papilloma and Epstein-Barr virus infections also play a role in the development of certain HNCs, particularly those in the oropharynx [4,5]. Although the prognosis has slightly improved over the past 30 years, the long-term survival rate is less than $50 \%$, and if cancer spreads, the prognosis could be fatal [4,5]. Currently, oral and laryngeal cancer treatment is predominantly based on the stage of the disease and requires combined approaches including surgery, radiotherapy, and cytotoxic chemotherapy [6]. Commonly used chemotherapy drugs are cisplatin (cDDP), carboplatin, docetaxel, and 5-flurouracil. In general, early-stage patients maintain speech and swallowing functions after treatment. While advanced-stages patients often suffer from permanent tracheostomy 
and natural voice loss. Chemotherapy is usually given before radiotherapy to shrink the tumor and make radiotherapy more effective. Following the discovery of the role of epidermal growth factor receptor (EGFR) signaling in development, progression, and prognosis of oral and laryngeal cancer, targeted molecular therapies have been developed including the EGFR monoclonal antibody cetuximab, approved by the FDA as a radiation sensitizer, and anti-EGFR tyrosine kinase inhibitors (erlotinib, gefitinib, etc.) [7].

Cisplatin-based chemotherapy is the mainstay therapy for cancers of the oral cavity and larynx. However, intrinsic or acquired drug resistance during treatment is the main limitation of its use [8]. Resistance mechanisms include changes in cellular uptake and efflux of cDDP, increased biotransformation and detoxification in the liver, and increased DNA repair and antiapoptotic mechanisms [8]. To overcome this problem, growing scientific interest has focused on the identification and development of powerful and bettertolerated treatments, including the use of natural compounds as adjuvants in combination therapy with conventional chemotherapy drugs. In this regard, combined therapy with S-adenosylmethionine (abbreviated as AdoMet, SAM or SAMe), a ubiquitous cofactor with pleiotropic effects on multiple cellular processes and the main biological methyl donor in transmethylation reactions, is emerging as a suitable strategy to improve the efficacy of standard chemotherapy and reduce both toxicity and side-effects $[9,10]$.

AdoMet has been extensively studied since its chemical structure was first described in 1952 [11]. A thorough review of the literature concerning the physiological and pathophysiological roles of AdoMet, among them clinical studies, highlights the therapeutic potential of this natural compound in the treatment of several diseases, including cancer [10,12].

Here, we summarize the structural, pharmacological, and clinical aspects of AdoMet and provide recent findings on the multitargeting antitumor activity of AdoMet in oral and laryngeal cancer cells, with particular emphasis on its molecular mechanisms and the promising chemoprotective and synergistic effects exerted in combination with cDDP and specific microRNAs.

\section{Structural, Metabolic and Regulatory Aspects}

AdoMet, together with its decarboxylated product S-adenosyl-(5')-3-methylthiopropilammine, is the only sulfonium compound found in mammalian tissues. The study of the biochemistry of AdoMet can be traced back to the experiments of Toennies [13], who first synthesized this compound allowing further successful characterization.

The positively charged sulfonium center makes AdoMet a chemically versatile molecule and the second most widely used enzyme substrate after ATP [13]. The chiral sulfur center of AdoMet exists in two enantiomeric forms, making AdoMet a diastereomer (Figure 1). The $(S, S)$ configuration represents the only biosynthesized form and also the biologically active form for almost all AdoMet-dependent methyltransferases [14]. Under physiological conditions or normal storage conditions, the absolute configuration $(S, S)$ racemizes to the enzymatically inactive $(R, S)$-AdoMet with a racemization rate of $8 \times 10^{-6} \mathrm{~S}^{-1}$ at $37^{\circ} \mathrm{C}$ pH $7.5[15]$.

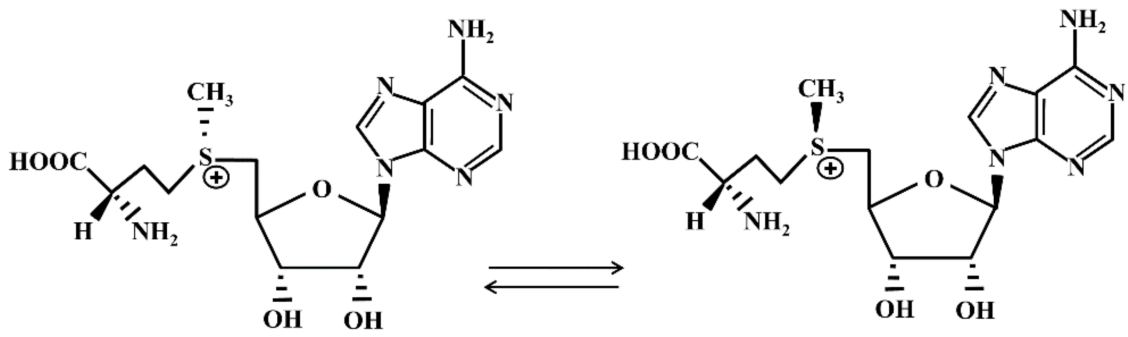

$(S, S)$ - S-Adenosyl-L-methionine

Figure 1. Conformations of S-adenosyl-L-methionine. 
AdoMet biosynthesis occurs mainly in the liver, where most of the methylation reactions take place [16-18], and is carried out by methionine adenosyltransferase (MAT) (EC 2.5.1.6), also known as AdoMet synthetase. This enzyme catalyzes the transfer of the adenosyl moiety of ATP to the sulfur atom of methionine in a two-step reaction requiring $\mathrm{Mg}^{2+}$ and $\mathrm{K}^{+}$ions following a $\mathrm{S}_{\mathrm{N}} 2$ mechanism [16-18]. Notably in this reaction, ATP plays an unusual role, undergoing complete dephosphorylation to provide energy for the removal of the reaction product from the enzyme active site. The biosynthesis of AdoMet is a process essential for life, as demonstrated by findings indicating that the gene encoding MAT protein represents one of the 482 genes necessary for the survival of all organisms and that its expression occurs in all cells except for some parasites and infectious agents which acquire AdoMet from their hosts [16-20].

AdoMet is a ubiquitous sulfurnucleoside widely known as the principal biological methyl donor for the majority of methyltransferases. Recently, AdoMet has been wellexplored as a therapeutic compound for its antiproliferation and proapoptotic effects in many types of human tumors [10,21-25].

Several multiple isoforms of MAT with different kinetic, regulatory, and physical properties have been characterized in mammalian tissues $[17,18]$. Recently, the presence of MAT in the nucleus has been demonstrated [26] and it has been hypothesized that this MAT activity might be involved in providing a source of nuclear AdoMet necessary for DNA or histone methylation. In mammals, three isoforms of AdoMet synthetase have been isolated and characterized, encoded by two different genes: MAT1A and MAT2A. $M A T 1 A$ is mostly expressed in the adult liver and encodes the $\alpha 1$ subunit, present in two isoforms: MAT III (dimer of $\alpha 1$ subunit) and MAT I (tetramer of $\alpha 1$ subunit). MAT2A instead encodes for the $\alpha 2$ catalytic subunit found in the MAT II isoenzyme, which is widely distributed and active in all cells with a marked predominance in the fetal liver, where it will be progressively replaced during development by the $M A T 1 A$ gene product $[17,18]$. The $M A T 2 B$ gene encodes a regulatory $\beta$ subunit existing in two distinct splicing variants: $\mathrm{V} 1$ and V2 that differ in NH2-terminal amino acid sequence of the protein. Both splicing variants of the $\beta$ regulatory subunit can be associated with MAT II $[17,18]$ and regulate its catalytic activity. The expression of the $M A T 2 B$ gene was observed only in mammals, especially in all examined extrahepatic tissues, and at low levels in the adult liver.

Over the past five decades, several studies have unequivocally established the significant role of AdoMet in the cellular metabolism of all living organisms, highlighting the importance of maintaining its physiological level in cells [27-29]. Notably, cells expressing the MAT1A gene show greater intracellular concentration of AdoMet than cells expressing $M A T 2 A$. Recent studies have shown that in the adult liver, the reduction in $M A T 1 A$ expression and the concomitant overexpression of MAT2A, known as "MAT1A/MAT2A switch", cause a chronic depletion of hepatic AdoMet levels, predisposing the liver to developing steatohepatitis, cirrhosis, and hepatocellular carcinoma [29].

The synthesis of AdoMet is part of the so-called one-carbon cycle that involves three interrelated biochemical pathways: the methionine cycle, the folate cycle, and the transsulfuration pathway. After transferring its methyl group to a large variety of acceptor substrates, AdoMet is converted to S-adenosylhomocysteine (AdoHcy or SAH), a strong competitive inhibitor of AdoMet-dependent methyltransferases, by the enzyme AdoHcy hydrolase (EC 3.3.1.1.), leading to the formation of adenosine and homocysteine (Hcy) through a hydrolytic reaction $[30,31]$. Hcy, in turn, will either be converted to cystathionine and then to glutathione (GSH) by trans-sulfuration, or will be remethylated to methionine by vitamin B12-dependent homocysteine methyltransferase through the transfer of a methyl group from the methyltetrahydrofolate, completing the methionine cycle (Figure 2) [30-32]. Modulation of the metabolic flux through the competing remethylation and trans-sulfuration pathways occurs at several levels. In the presence of low levels of AdoMet, Hcy metabolism is shifted towards remethylation to preserve methionine and AdoMet synthesis. On the other hand, high AdoMet levels and methionine excess trigger the trans-sulfuration 
pathway. In this light, AdoMet is able to control methionine metabolism in response to impairments of physiological conditions.

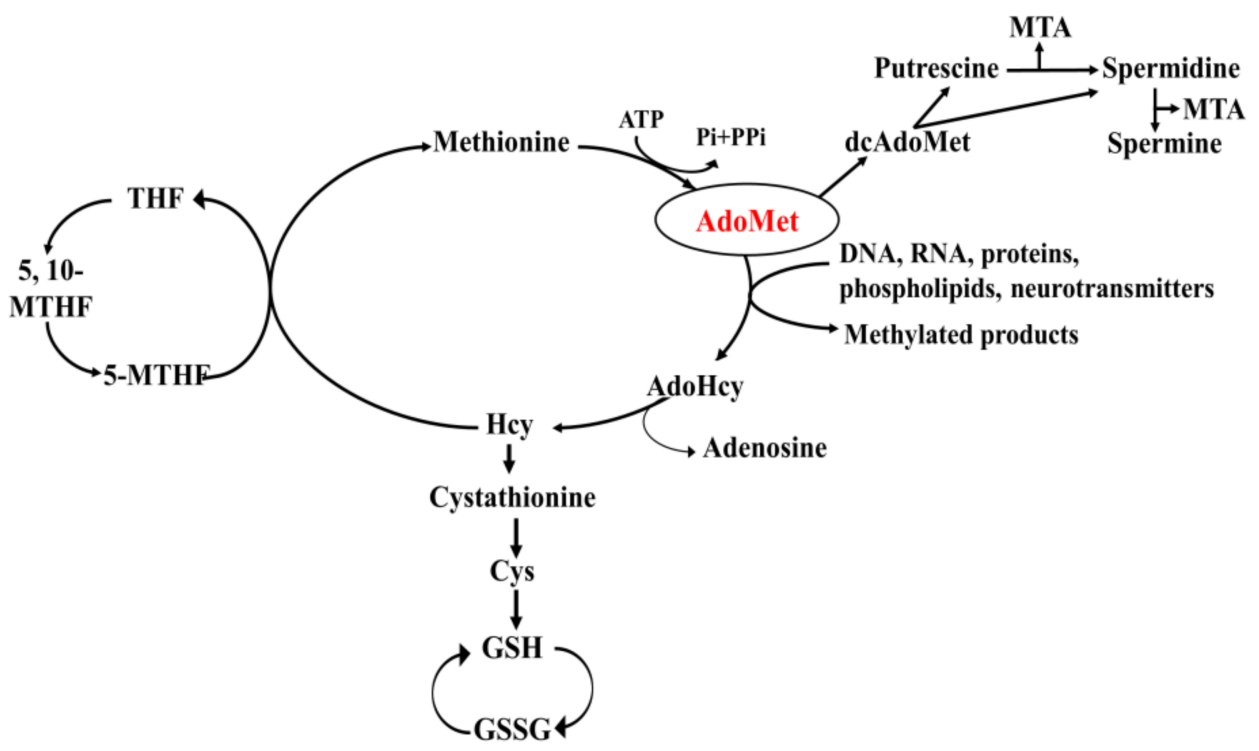

Figure 2. Metabolic relationships among transmethylation, trans-sulfuration, folate cycle, and polyamine synthesis. AdoHcy: S-adenosylhomocysteine; dcAdoMet: S-adenosyl-(5')-3-methylthiopropylamine; Cys: cysteine; GSH: reduced glutathione; GSSG: oxidized gluthatione: Hcy: homocysteine; MTA: 5'deoxy-5'-methylthioadenosine; 5-MTHF: 5-methyltetrahydrofolate; THF: tetrahydrofolate; 5,10-MTHF: 5,10-methylenetetrahydrofolate.

AdoMet exerts a primary role in cellular metabolism through a variety of welldocumented biological functions. The reactivity of AdoMet is based on the high-energy sulfonium ion that makes the molecule an efficient methyl-, aminopropyl-, and adenosylgroup donor $[9,12,30-32]$, as well as a key player in three important metabolic pathways, such as transmethylation, trans-sulfuration, and polyamine biosynthesis.

The first known and well-studied metabolic reaction from the elucidation of the AdoMet structure is the transmethylation pathway, in which the sulfonium compound donates its methyl group to a large variety of acceptor molecules in reactions catalyzed by methyltransferases [31]. Epigenetic modification is the most important effect obtained by the methylation reaction, and represents one of the most studied processes in cancer. Experimental evidence has been reported, showing that AdoMet, through an epigenetic mechanism, is able to regulatethe expression of genes playing a crucial role in cell migration, invasion, and metastasis [33-36]. In recent decades, many in vitro and in vivo studies have shown the involvement of AdoMet in various cellular processes, including proliferation, differentiation, cell cycle regulation, and apoptosis, demonstrating that the sulfonium compound exerts pleiotropic effects on signal transduction in different cell types and that it is able to halt the progression of many human tumors [21-25,33-36].

In addition to its well-known functions as a methyl donor and precursor of GSH and polyamine biosynthesis, the sulfonium compound serves as a source of 5'-deoxyadenosyl radicals in anaerobic organisms [37]. Radical AdoMet enzymes are found in all domains of life. These enzymes share a CX3CX2C motif forming a characteristic [4Fe-4S] cluster that binds AdoMet and catalyzes its reductive cleavage to generate [4Fe-4S]-methionine and a 5 -deoxyadenosyl 5'-radicals. These radical intermediates are highly reactive and participate in a wide variety of reactions involved in DNA repair, RNA and protein modification, and the biosynthesis of vitamins, coenzymes, and natural products. In the last decade, more than 40 distinct biochemical transformations regulated by AdoMet radical enzymes have been discovered, although their involvement in biochemical processes has not yet been fully understood [37]. Moreover, AdoMet is involved in diphthamide, ethylene, and 
biotin biosynthesisand in post-translational modifications during t-RNA biosynthesis, and acts as an allosteric modulator of numerous enzymatic systems.

As a result of its many metabolic and regulatory roles, a variety of pharmacological effects are attributed to AdoMet, including anti-inflammatory, analgesic, and antidepressive effects, as well as a therapeutic potential in the treatment of liver diseases.

\section{Pharmacological and Clinical Aspects}

Owing to its crucial role in multiple biochemical pathways, AdoMet has been intensively studied as a potential therapeutic agent for the treatment of various clinical disorders. AdoMet is commercially available worldwide, both as nutraceutical supplement and as a pharmaceutical product, depending on the different dosage and different local laws. AdoMet is used as an antidepressant in some European countries (e.g., Italy) under the marketing name Samyr, while in the United States and Canada it is only utilized as a dietary supplement under the marketing name SAM-e or SAMe. In Russia, China, and South America, it is widely used in chronic liver diseases and cholestasis. AdoMet is an FDA-approved dietary supplement and pharmaceutical preparations of this compound are available as intravenous, intramuscular, and oral forms. Notably, exogenous AdoMet is generally well-tolerated and able to cross the intestinal wall, increasing its plasma concentrations. Maximum plasma concentrations of $0.5-1 \mathrm{mg} / \mathrm{L}$ are achieved 3-5 h after doses of AdoMet ranging from 400 to $1000 \mathrm{mg} /$ day [38]. These high doses are required to overcome the poor oral bioavailability and rapid metabolism of AdoMet that, despite its high solubility, is characterized by low cellular permeability and high chemical instability [39]. However, salts of AdoMet with strong anions can provide stability to the molecule, and at present, only sulfuric acid, 1,4-butanedisulfonic acid, and p-toluenesulfonic acid (tosylated)(PTS) salts are available on the market. Recently, a new formulation of AdoMet phytate showed better stability and pharmacokinetic properties in comparison with the standard PTS salt [40]. AdoMet in the form of its stable p-toluensulphonate or butanedisulfonate salt has been used for more than 30 years in the treatment of depression, liver disorders, musculoskeletal and joint disorders such as, osteoarthritis, fibromyalgia, neurodegenerative disorders, and more remarkably, major depressive disorders (MDD) (Figure 3) [41].

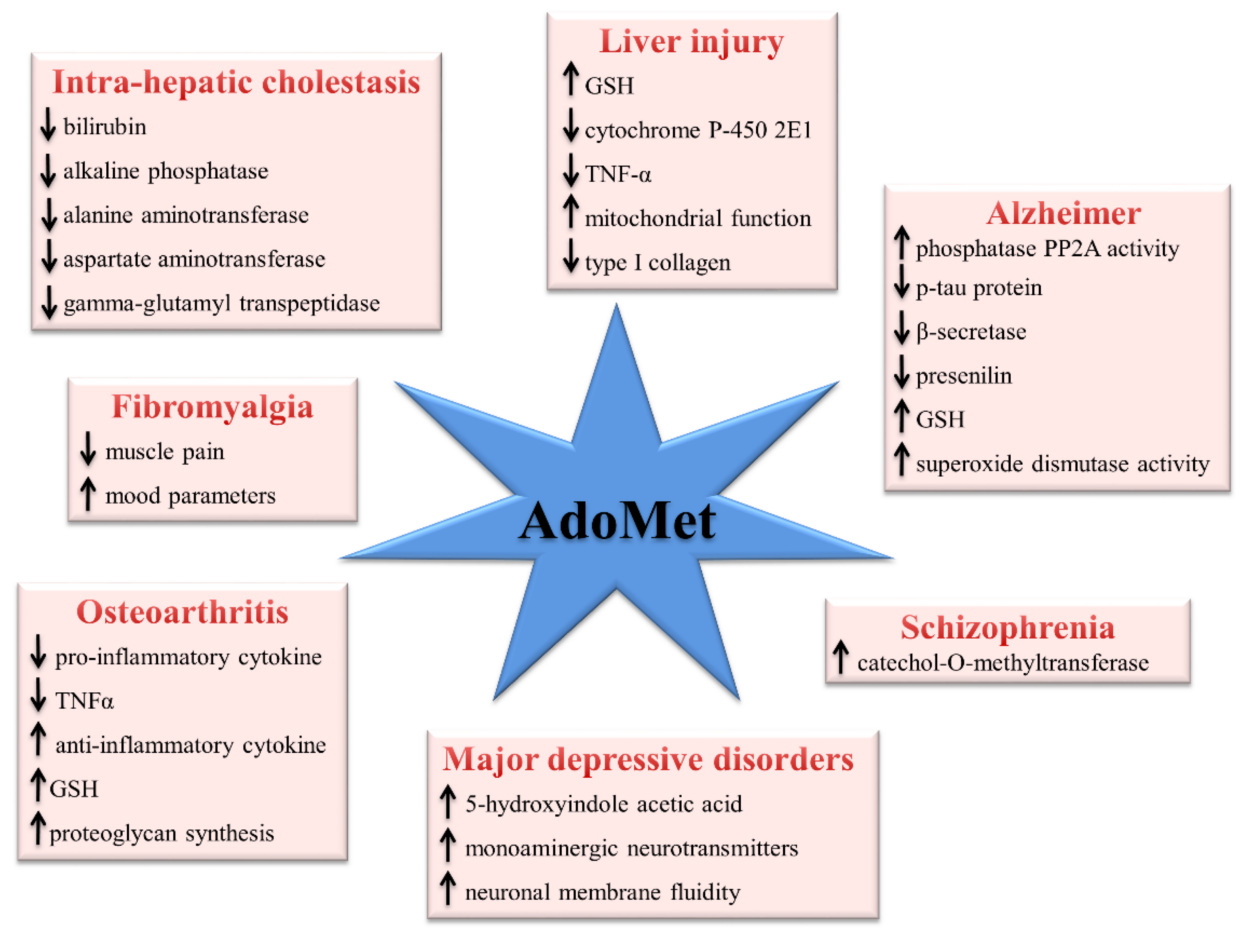

Figure 3. AdoMet's ability to counteract the pathological hallmarks of many human diseases by different mechanisms. 
AdoMet is a major endproduct of the one-carbon metabolism, a complex metabolic route involving folate and methionine cycles that generates and coordinates several final outputs including the substrate for methylation reactions and the maintenance of redox balance. Changes in one-carbon metabolism caused by nutritional or genetic alteration of the enzymes involved and the decrease in AdoMet/AdoHcy ratio due to accumulation of AdoHcy, the strong competitive inhibitor of AdoMet-dependent methyltransferases, may explain the aberrant methylation that is implicated as a pathogenic mechanism in psychiatric and neurological diseases, including MDD and Alzheimer's disease [42].

Although the mechanism of antidepressant activity of AdoMet is not well-elucidated, the available evidence suggests that AdoMet functions as a methyl-donating cofactor in the biosynthesis of monoamine neurotransmitters, such as norepinephrine, dopamine, and serotonin, which play important roles in maintaining a normal mood. In addition, AdoMet treatment increases the concentrations of 5-hydroxyindole acetic acid, a marker for serotoninergic activity. On the contrary, low levels of AdoMet may negatively modulate some critical components of monoaminergic neurotransmitters. Interestingly, low levels of AdoMet have been detected in serum and cerebrospinal fluid of patients with depressive disorders [43] while AdoMet supplementation was found to enhance its levels in cerebrospinal fluid, demonstrating that the sulfonium compound is able to cross the blood-brain barrier [44].

It has been proposed that the antidepressant role of AdoMet could also be related to the stimulatory effect exerted on membrane phospholipid methylation, a process involved in the promotion of the fluidity of neuronal membrane and in the enhancement of its dynamic functions, including induction of monoamine receptors and monoamine transporters and receptor coupling, thus maintaining adequate neurotransmission and preventing depression [45]. On this basis, supplementation with the methyl donor AdoMet has been proposed as a potential therapeutic approach in patients with MDD [46].

DNA methylation and other epigenetic factors are important in the pathogenesis of Alzheimer's disease, the most common neurodegenerative disorder of age-derived diseases characterized by deposits of $\beta$-amyloid protein and intracellular fibrillar deposits of hyperphosphorylated tau protein leading to neuronal death and brain atrophy. It has been recently reported that alteration of one-carbon metabolism regulates the expression of two key enzymes in the amyloid pathway, such as, $\beta$-secretase and presenilin, and that low methylation index is associated with increased $\mathrm{A} \beta$ product. Moreover, supplementation with AdoMet stabilizes the heterotrimeric form of phosphatase PP2A, resulting in an increased enzymatic activity, a reduced level of pathological tau protein, and improved cognition. Dysregulation of one-carbon metabolism leads not only to alteration of the methylation cycle with increased Hcy levels and AdoHcy accumulation, but it is also strictly connected with alteration of GSH metabolism consequent to impairment of transsulfuration. AdoMet prevents oxidative stress associated with one-carbon metabolism alteration decreasing lipid peroxidation levels through modulating GSH metabolism and superoxide dismutase activity, suggesting that DNA hypomethylation and alteration of the redox homeostasis are related and could represent the mechanisms through which dysregulation of one-carbon metabolism is involved in brain diseases [45,47]. Several preclinical and clinical data have highlighted the association between schizophrenia symptoms, such as aggressiveness, and increased levels of dopaminergic activity in the mesolimbic areas of the brain. Furthermore, several hypoactive variants of catechol-O-methyltransferase (COMT), the enzyme responsible for dopamine degradation, have been reported to be linked to aggressive behaviors in schizophrenia patients. The few clinical studies in literature indicate that AdoMet is able to decrease aggressive behavior in schizophrenia through increased COMT activity $[48,49]$.

The therapeutic potential of AdoMet in treating osteoarthritis was revealed in the first clinical trials utilizing AdoMet for depression, which evidenced that patients receiving AdoMet saw benefits intheir osteoarthritic symptoms. Since then, trials involving more than 22,000 participants have confirmed the therapeutic efficacy of AdoMet against osteoarthritis, 
a severe debilitating degenerative joint disease resulting from the breakdown of joint cartilage and underlying bone [50]. Available evidence indicates that AdoMet exerts anti-inflammatory effects by reducing the expression of the proinflammatory cytokine tumor necrosis factor-alpha and by increasing the expression of the anti-inflammatory cytokine interleukin-10. It has been suggested that AdoMet's anti-inflammatory activity is mediated in part by the modulation of methylation and/or binding of histones to the promoter region of proinflammatory or anti-inflammatory cytokine genes [51]. The exact mechanism of AdoMet in slicing symptoms of osteoarthritis is unknown, but evidence suggests that it may play a role in reducing inflammation and increasing proteoglycan synthesis. Interestingly, AdoMet participates in the synthesis of polyamines that might stabilize and protect these polyanionic macromolecules from damage caused by proteolytic and glycolytic enzymes. AdoMet protects synovial cells by inhibiting GSH depletion and supporting GSH peroxidase levels. Finally, AdoMet reduces osteoarthritic pain by acting as an analgesic agent [52,53]. Clinical studies have highlighted that AdoMet shows greater action, more tolerated by the body and sustained for a longer period, than nonsteroidal anti-inflammatory drugs.

In fibromyalgia, a nonarticular rheumatic disorder characterized by generalized musculoskeletal pain, stiffness, fatigue, and sleep disturbances, AdoMet treatment has shown beneficial effects on muscle pain and mood parameters, significantly reducing primary symptom outcomes of pain [46,54].

The liver is the major site of AdoMet synthesis and degradation, and it is vital for maintaining AdoMet homeostasis. AdoMet is used for intrahepatic cholestasis treatment, as well as for curing chronic liver diseases due to alcohol abuse. Clinical studies have shown that after AdoMet administration to patients with intrahepatic cholestasis, the liver functions were significantly ameliorated. Serum level of total bilirubin, alkaline phosphatase, alanine aminotransferase, aspartate aminotransferase, and gamma-glutamyl transpeptidase were significantly reduced within the third week of treatment, and came back to normal levels after four weeks, demonstrating the cytoprotective action of AdoMet in acute and chronic liver injury [55]. Furthermore, it has been shown that patients with cirrhosis had decreased MAT1A expression with consequent accumulation of methionine and significantly reduced AdoMet levels [28,29,38,56]. The physiological roles of the sulfonium compound in liver and its therapeutic use in liver disease are reviewed in-depth in the literature [16-18,28,29,38,55,56].

To confirm these positive clinical outcomes and validate new therapies, more studies with a larger population and longer treatments are needed. Due to its involvement in many physiological and pathological processes, AdoMet can be considered a structural model to build potential new inhibitors for pharmacological intervention.

\section{Anticancer Effects of the Methyl Donor S-adenosylmethionine in Oral and Laryngeal Cancer: Mechanisms, Molecular Targets, and Potential Therapeutic Applications}

Over the past decade, many in vitro and in vivo studies have highlighted the antiproliferative, proapoptotic, and antimetastatic effects of AdoMet in different types of human cancers [21-25]. Our research group thoroughly investigated the anticancer effects of AdoMet in oral and laryngeal squamous cell carcinoma (OLSCC) and has provided to the literature the first and so far only experimental evidence on the underlying mechanisms involving the modulation of a broad spectrum of signal transduction pathways [57-60]. AdoMet exerts antiproliferative activities in HNSCC through well-documented effects on a variety of biological processes, including cell growth suppression, induction of cell death by apoptosis and autophagy, activation of ER-stress, and inhibition of cell migration and invasion [57]. AdoMet slows down cancer cell proliferation by causing G2/Mand S-phase arrest in Cal-33 and JHU-SCC-011 cells, respectively. At a molecular level AdoMet downregulates the expression of cyclin D1, E1, A2, B1, and p21 cell-cycle inhibitor, leaving p53 unmodified, indicating that the cell-cycle arrest induced by AdoMet is a p53/p21-independent process. Interestingly, in Cal-33 cells, the effect of AdoMet is medi- 
ated by upregulation of phosho-cdc25C, a phosphatase that regulates cell-cycle progression in $\mathrm{S}$ phase and mitosis by removing inhibitory phosphate groups from cyclin-dependent kinases [58], providing evidence that in OLSCC the AdoMet-dependent phosphorylation of cdc25C acts as a metabolic checkpoint for the G2/M phase transition. This regulatory mechanism is p53-independent and is therefore particularly relevant for the DNA damage response in cancer cells characterized by high levels of mutant p53, such as in Cal-33.

AdoMet induces apoptosis viathe mitochondrial pathway through activating caspase cascade paralleled by increased $\mathrm{Bax} / \mathrm{Bcl}-2$ ratio, and triggers autophagic cell death with a consistent increase in the level of the autophagic marker LC3B-II. In OLSCC, AdoMet, for the first time, was found to induce ERstress and activate the unfolded protein response (UPR) signaling, resulting in the upregulation of CHOP and XBP1, crucial markers of ER-stress-mediated apoptotic pathway, and in phosphorylation and activation of mitogenactivated protein kinases. Among these, JNK has been found to play a crucial role in ER-stress-induced autophagy through Bcl-2 phosphorylation, which prevents this protein interacting and inhibiting beclin-1, the crucial autophagy regulator [61]. The ability of AdoMet to trigger pro-death activity of UPR while simultaneously inhibiting its prosurvival function represents one of the anticancer mechanisms utilized by AdoMet that is very promising in terms of potential therapeutic outcome.

The antimetastatic properties of AdoMet have been well-documented in different types of human cancers, and the underlying mechanisms ranging from epigenetic regulation of genes responsible for cell migration and invasion [33-36] to the modulation of specific microRNAs (miRNAs) [59,62,63], have been elucidated. In OLSCC, AdoMet inhibits the migration and invasion of Cal-33 and JHU-SCC-011 cells by downregulating the expression of MMP2 and MMP9, two metalloproteinases involved in the degradation of the basement membrane and extracellular matrix.

EMT, a highly regulated transdifferentiation process enablingcancer cells to acquire migratory and invasive properties as well as resistance to apoptosis and drug treatments, has been shown to play a key role in HNSCC cancer progression and metastasis. Therefore, targeting EMT might offer promising opportunities to restrain metastatic spreading and related mortality in these cancer cells [64]. AdoMet was found to modulate EMT in OLSCC by decreasing the mesenchymal markers $\mathrm{N}$-cadherin and vimentin and concomitantly increasing the epithelial marker E-cadherin, indicative of the ability of AdoMet to reverse the EMT process. Moreover, AdoMet downregulates AKT, $\beta$-catenin, SMAD-2/3, and their phosphorylated forms, indicating that TGF- $\beta / \mathrm{SMAD} / \beta$-catenin signaling represents the target selected by AdoMet to modulate EMT and cell motility and invasiveness.

The increasing incidence of resistance to chemotherapeutic agents has become a major issue in the treatment of oral cancer. The ability of AdoMet to act as a chemosensitizer has been amply documented in several types of human cancers $[10,21,65]$. Our research group demonstrated that in head and neck squamous cancer cells, the combination of AdoMet and cisplatin produced a synergism as evidenced by the value of the combination index (CI) and enhanced apoptosis by strongly activating caspase cascade and PARP cleavage with a concomitant increase in Bax/Bcl-2 ratio [57,60]. The combined treatment also potentiated the activation of JNK and ERK1/2 and downregulated the expression level of Bcl-2, thus modulating two important signaling pathways involved in cDDP resistance. AdoMet has proven to be very effective in enhancing cDDP anticancer effects, reducing the drug concentration needed to induce apoptosis and to inhibit migration to the extremely low value of $0.18 \mu \mathrm{M}$ [57-60]. Notably, this subtoxic cDDP concentration is one of the lowest reported so far in combination treatments with natural chemosensitizer compounds, highlighting AdoMet as an effective adjuvant to be used in combination therapy to overcome drug resistance in OLSCC. EMT has attracted a lot of attention in recent years with regard to its relationship with the mechanism of chemotherapy drug resistance in oral cancer [66]. In line with this evidence, the inhibition of EMT by AdoMet could represent an effective mechanism to overcome resistance to cDDP, further stressing the potential therapeutic utility of the sulfonium compound. 
More and more evidence has shown that epigenetic modulation of the level of noncoding RNA involved in oncogenic functions represents one of the mechanisms underlying the anticancer activity of AdoMet. Noncoding RNAs, especially miRNAs, have a significant role in carcinogenesis of head and neck cancers, acting both as oncogenes to promote cancer development and invasion and as tumor suppressors by participating in the modulation of cell proliferation, differentiation, migration, and invasion [63]. Notably, the role of miRNAs as biomarkers in HNSCC has recently been evidenced [67]. In laringeal squamous cancer cells (LSCC), AdoMet strongly reduced the level of miR-888-5p, an oncogenic miRNA involved in the development and maintenance of the tumor cell phenotype. Cellular treatment with AdoMet in combination with miR-888-5p inhibitor synergistically potentiated apoptosis, inhibited tumor cell migration induced by the sulfonium compound and also induced the upregulation, both at transcriptional and at traductional level, of c-Myc-binding protein (MYCBP) and E-cadherin (CDH1), indicating that the proapoptotic and antimetastatic activity of AdoMet in LSCC is mediated by the downregulation of the oncogenic miR-888-5p and that MYCBP and CDH1 play a role in the mechanism underlying the anticancer activity of AdoMet in LSCC.

Figure 4 schematically summarizes the anticancer activity of AdoMet in OLSCC.

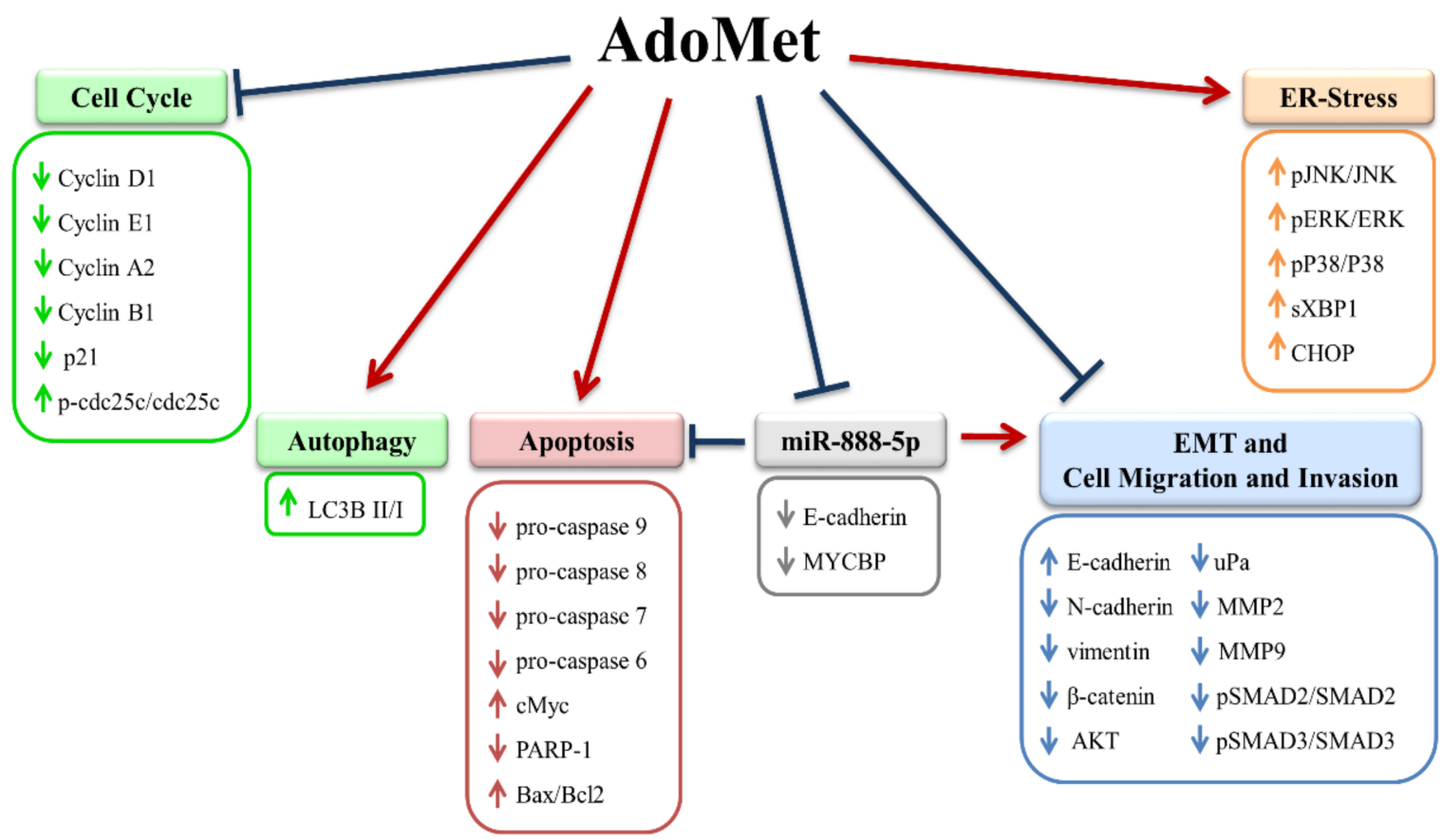

Figure 4. Schematic representation summarizing the multitargeted anticancer effects of AdoMet in OSLCC.

In conclusion, the identification and development of new compounds with antitumor activity, and at the same time, with little or no toxicity,is necessary to improve the therapies of patients with cancers of the oral cavity and larynx. Overall, the data reported in this review underline that AdoMet can be considered a promising agent for adjuvant therapy of OLSCC by virtue of its ability to inhibit cell proliferation, migration, and invasion and to activate tightly regulated cell-death mechanisms. The potential of AdoMet to sensitize OLSCC to CDDP by reversing chemoresistance together with the ability to downregulate oncogenic miRNAs, such as miR-888-5p, open new opportunities in the field of oral and laryngeal cancer therapy based on combined treatments aimed at inducingsynergistic anticancer effects. 
Author Contributions: L.M., G.C. and M.P. (Marina Porcelli) conceived the idea and wrote the manuscript; A.C., R.V.T., R.G., M.P. (Martina Pagano) and F.V. contributed to the literature review and writing. All authors have read and agreed to the published version of the manuscript.

Funding: This research received no external funding.

Conflicts of Interest: The authors declare no conflict of interest.

\section{References}

1. Chow, L.Q.M. Head and neck cancer. N. Engl. J. Med. 2020, 382, 60-72. [CrossRef] [PubMed]

2. Johnson, D.E.; Burtness, B.; Leemans, C.R.; Lui, V.W.Y.; Bauman, J.E.; Grandis, J.R. Head and neck squamous cell carcinoma. Nat. Rev. Dis. Primers 2020, 6, 92. [CrossRef] [PubMed]

3. Marur, S.; Forastiere, A.A. Head and neck squamous cell carcinoma: Update on epidemiology, diagnosis, and treatment. Mayo. Clin. Proc. 2016, 91, 386-396. [CrossRef] [PubMed]

4. Maasland, D.H.; Van den Brandt, P.A.; Kremer, B.; Goldbohm, R.A.; Schouten, L.J. Alcohol consumption, cigarette smoking and the risk of subtypes of head-neck cancer: Results from the Netherlands Cohort Study. BMC Cancer 2014, 14, 187. [CrossRef] [PubMed]

5. Hashim, D.; Genden, E.; Posner, M.; Hashibe, M.; Boffetta, P. Head and neck cancer prevention: From primary prevention to impact of clinicians on reducing burden. Ann. Oncol. 2019, 30, 744-756. [CrossRef] [PubMed]

6. Kulkarni, P.; Saxena, U. Head and neck cancers, the neglected malignancies: Present and future treatment strategies. Expert Opin. Ther. Targets 2014, 18, 351-354. [CrossRef] [PubMed]

7. Kitamura, N.; Sento, S.; Yoshizawa, Y.; Sasabe, E.; Kudo, Y.; Yamamoto, T. Current trends and future prospects of molecular targeted therapy in head and neck squamous cell carcinoma. Int. J. Mol. Sci. 2021, 22, 240. [CrossRef]

8. Chen, S.H.; Chang, J.Y. New insights into mechanisms of cisplatin resistance: From tumor cell to microenvironment. Int. J. Mol. Sci. 2019, 20, 4136. [CrossRef]

9. Lu, S.C. S-Adenosylmethionine. Int. J. Biochem. Cell Biol. 2000, 32, 391-395. [CrossRef]

10. Mosca, L.; Vitiello, F.; Coppola, A.; Borzacchiello, L.; Ilisso, C.P.; Pagano, M.; Caraglia, M.; Cacciapuoti, G.; Porcelli, M. Therapeutic potential of the natural compound S-adenosylmethionine as a chemoprotective synergistic agent in breast, and head and neck cancer treatment: Current status of research. Int. J. Mol. Sci. 2020, 21, 8547-8561. [CrossRef]

11. Cantoni, G.L. S-Adenosylmethionine, a new intermediate formed enzimatically from L-metionine and adenosine triphosphate. J. Biol. Chem. 1953, 203, 403-416. [CrossRef]

12. Fontecave, M.; Atta, M.; Mulliez, E. S-Adenosylmethionine: Nothing goes to waste. Trends Biochem. Sci. 2004, 29, 243-249. [CrossRef] [PubMed]

13. Toennies, G. Sulfonium reactions of methionine and their possible metabolic significance. J. Biol. Chem. 1940, 132, 455. [CrossRef]

14. Zhang, J.; Klinman, J.P. High-performance liquid chromatography separation of the (S,S)- and (R,S)-forms of S-adenosyl-Lmethionine. Anal. Biochem. 2015, 476, 81-83. [CrossRef] [PubMed]

15. Hoffman, J.L. Chromatographic analysis of the chiral and covalent instability of S-adenosyl-L-methionine. Biochemistry 1986, 25, 4444-4449. [CrossRef]

16. Murray, B.; Barbier-Torres, L.; Fan, W.; Mato, J.M.; Lu, S.C. Methionine adenosyltransferases in liver cancer. World J. Gastroenterol. 2019, 25, 4300-4319. [CrossRef]

17. Ramani, K.; Lu, S.C. Methionine adenosyltransferases in liver health and diseases. Liver Res. 2017, 1, 103-111. [CrossRef]

18. Maldonado, L.Y.; Arsene, D.; Mato, J.M.; Lu, S.C. Methionine adenosyltransferases in cancers: Mechanisms of dysregulation and implications for therapy. Exp. Biol. Med. 2018, 243, 107-117. [CrossRef]

19. Minici, C.; Mosca, L.; Ilisso, C.P.; Cacciapuoti, G.; Porcelli, M.; Degano, M. Structures of catalytic cycle intermediates of the Pyrococcus furiosus methionine adenosyltransferase demonstrate negative cooperativity in the archaeal orthologues. J. Struct. Biol. 2020, 210, 107462. [CrossRef]

20. Porcelli, M.; Ilisso, C.P.; Mosca, L.; Cacciapuoti, G. A thermostable archaeal S-Adenosylmethionine synthetase: A promising tool to improve the synthesis of Adenosylmethionine analogs of biotechnological interest. Bioengineered 2015, 6, 184-186. [CrossRef]

21. Mosca, L.; Pagano, M.; Pecoraro, A.; Borzacchiello, L.; Mele, L.; Cacciapuoti, G.; Porcelli, M.; Russo, G.; Russo, A. S-Adenosyl-LMethionine overcomes uL3-mediated drug resistance in p53 deleted colon cancer cells. Int. J. Mol. Sci. 2020, 22, 103. [CrossRef] [PubMed]

22. Ilisso, C.P.; Delle Cave, D.; Mosca, L.; Pagano, M.; Coppola, A.; Mele, L.; Caraglia, M.; Cacciapuoti, G.; Porcelli, M. Sadenosylmethionine regulates apoptosis and autophagy in MCF-7 breast cancer cells through the modulation of specific microRNAs. Cancer Cell Int. 2018, 188, 197. [CrossRef] [PubMed]

23. Mahmood, N.; Cheishvili, D.; Arakelian, A.; Tanvir, I.; Khan, H.A.; Pépin, A.S.; Szyf, M.; Rabbani, S.A. Methyl donor Sadenosylmethionine (SAM) supplementation attenuates breast cancer growth, invasion and metastasis in vivo; therapeutic and chemopreventive applications. Oncotarget 2018, 9, 5169-5183. [CrossRef] [PubMed]

24. Cave, D.D.; Ilisso, C.P.; Mosca, L.; Pagano, M.; Martino, E.; Porcelli, M.; Cacciapuoti, G. The anticancer effects of Sadenosylmethionine on breast cancer cells. JSM Chem. 2017, 5, 1049. 
25. Delle Cave, D.; Desiderio, V.; Mosca, L.; Ilisso, C.P.; Mele, L.; Caraglia, M.; Cacciapuoti, G.; Porcelli, M. S-Adenosylmethioninemediated apoptosis is potentiated by autophagy inhibition induced by chloroquine in human breast cancer cells. J. Cell. Physiol. 2017, 233, 1370-1383. [CrossRef]

26. Hao, X.; Zhou, M.; Li, H.; Angres, I.A. Novel immunoassays to detect methionine adenosyltransferase activity and quantify S-adenosylmethionine. FEBS Lett. 2017, 591, 1114-1125. [CrossRef]

27. Jung, Y.S. Metabolism of sulfur-containing amino acids in the liver: A link between hepatic injury and recovery. Biol. Pharm. Bull. 2015, 38, 971-974. [CrossRef]

28. Mora, S.I.; García-Román, J.; Gómez-Ñañez, I.; García-Román, R. Chronic liver diseases and the potential use of S-Adenosyl-Lmethionine as a hepato protector. Eur. J. Gastroenterol. Hepatol. 2018, 30, 893-900. [CrossRef]

29. Mato, M.J.; Corrales, F.J.; Lu, S.C.; Avila, M.A. S-Adenosylmethionine: A control switch that regulates liver function. FASEB J. 2002, 16, 15-26. [CrossRef]

30. Froese, D.S.; Fowler, B.; Baumgartner, M.R. Vitamin B12, folate, and the methionine remethylation cycle-biochemistry, pathways, and regulation. J. Inherit. Metab. Dis. 2019, 42, 673-685. [CrossRef]

31. Chiang, P.K.; Gordon, R.K.; Tal, J.; Zeng, G.C.; Doctor, B.P.; Pardhasaradhi, K.; McCann, P.P. S-Adenosylmethionine and methylation. FASEB J. 1996, 10, 471-480. [CrossRef] [PubMed]

32. Lu, S.C. Regulation of glutathione synthesis. Mol. Aspects Med. 2009, 30, 42-59. [CrossRef] [PubMed]

33. Pakneshan, P.; Szyf, M.; Farias-Eisner, R.; Rabbani, S.A. Reversal of the hypomethylation status of urokinase (uPA) promoter blocks breast cancer growth and metastasis. J. Biol. Chem. 2004, 279, 31735-31744. [CrossRef] [PubMed]

34. Shukeir, N.; Pakneshan, P.; Chen, G.; Szyf, M.; Rabbani, S.A. Alteration of the methylation status of tumor-promoting genes decreases prostate cancer cell invasiveness and tumorigenesis in vitro and in vivo. Cancer Res. 2006, 66, 9202-9210. [CrossRef]

35. Hussain, Z.; Khan, M.I.; Shahid, M.; Almajhdi, F.N. S-adenosylmethionine, a methyl donor, up regulates tissue inhibitor of metalloproteinase-2 in colorectal cancer. Genet. Mol. Res. 2013, 12, 1106-1118. [CrossRef]

36. Parashar, S.; Cheishvili, D.; Arakelian, A.; Hussain, Z.; Tanvir, I.; Khan, H.A. S-Adenosylmethionine blocks osteosarcoma cells proliferation and invasion in vitro and tumor metastasis in vivo: Therapeutic and diagnostic clinical applications. Cancer Med. 2015, 4, 732-744. [CrossRef]

37. Broderick, W.E.; Hoffman, B.M.; Broderick, J.B. Mechanism of radical initiation in the radical S-Adenosyl-l-methionine superfamily. Acc. Chem. Res. 2018, 51, 2611-2619. [CrossRef]

38. Lu, S.C.; Mato, J.M. S-Adenosylmethionine in liver health, injury, and cancer. Physiol. Rev. 2012, 92, 1515-1542. [CrossRef]

39. Yang, J.; He, Y.; Du, Y.X.; Tang, L.L.; Wang, G.J.; Fawcett, J.P. Pharmacokinetic properties of S-Adenosylmethionine after oral and intravenous administration of its tosylate disulfate salt: A multiple-dose, open-label, parallel group study in healthy Chinese volunteers. Clin. Ther. 2009, 31, 311-320. [CrossRef]

40. Francioso, A.; Fanelli, S.; d'Erme, M.; Lendaro, E.; Miraglia, N.; Fontana, M.; Cavallaro, R.A.; Mosca, L. Pharmacokinetic properties of a novel formulation of S-adenosyl-L-methionine phytate. Amino Acids 2021, 53, 1559-1568. [CrossRef]

41. De Berardis, D.; Orsolini, L.; Serroni, N.; Girinelli, G.; Iasevoli, F.; Tomasetti, C.; De Bartolomeis, A.; Mazza, M.; Valchera, A.; Fornaro, M.; et al. A comprehensive review on the efficacy of S-Adenosyl-L-methionine in Major Depressive Disorder. CNS Neurol. Disord. Drug Targets 2016, 15, 35-44. [CrossRef]

42. Ducker, G.S.; Rabinowitz, J.D. One-carbon metabolism in health and disease. Cell Metab. 2017, 25, 27-42. [CrossRef] [PubMed]

43. Bottiglieri, T. Isocratic high performance liquid chromatographic analysis of S-adenosylmethionine and S-adenosylhomocysteine in animal tissues: The effect of exposure to nitrous oxide. Biomed. Chromatogr. 1990, 4, 239-241. [CrossRef] [PubMed]

44. Sharma, A.; Gerbarg, P.; Bottiglieri, T.; Massoumi, L.; Carpenter, L.L.; Lavretsky, H.; Muskin, P.R.; Brown, R.P.; Mischoulon, D.; as Work Group of the American Psychiatric Association Council on Research. S-Adenosylmethionine (SAMe) for neuropsychiatric disorders: A clinician-oriented review of research. J. Clin. Psychiatry 2017, 78, e656-e667. [CrossRef]

45. Gao, J.; Cahill, C.M.; Huang, X.; Roffman, J.L.; Lamon-Fava, S.; Fava, M.; Mischoulon, D.; Rogers, J.T. S-Adenosyl Methionine and transmethylation pathways in neuropsychiatric diseases throughout life. Neurotherapeutics 2018, 15, 156-175. [CrossRef] [PubMed]

46. Alvarez-Mon, M.A.; Ortega, M.A.; García-Montero, C.; Fraile-Martinez, O.; Monserrat, J.; Lahera, G.; Mora, F.; Rodriguez-Quiroga, A.; Fernandez-Rojo, S.; Quintero, J.; et al. Exploring the role of nutraceuticals in major depressive disorder (MDD): Rationale, state of the art and future prospects. Pharmaceuticals 2021, 14, 821. [CrossRef] [PubMed]

47. Beauchamp, L.C.; Liu, X.M.; Sedjahtera, A.; Bogeski, M.; Vella, L.J.; Bush, A.I.; Adlard, P.A.; Barnham, K.J. S-Adenosylmethionine rescues cognitive deficits in the rTg4510 animal model by stabilizing protein phosphatase $2 \mathrm{~A}$ and reducing phosphorylated Tau. J. Alzheimer's Dis. 2020, 77, 1705-1715. [CrossRef]

48. Tsao, D.; Diatchenko, L.; Dokholyan, N.V. Structural mechanism of S-Adenosylmethionine binding to catechol Omethyltransferase. PLoS ONE 2011, 6, e24287. [CrossRef]

49. Strous, R.D.; Ritsner, M.S.; Adler, S.; Ratner, Y.; Maayan, R.; Kotler, M.; Lachman, H.; Weizman, A. Improvement of aggressive behavior and quality of life impairment following S-Adenosyl-methionine (SAM-e) augmentation in schizophrenia. Eur. Neuropsychopharmacol. 2009, 19, 14-22. [CrossRef]

50. Latourte, A.; Kloppenburg, M.; Richette, P. Emerging pharmaceutical therapies for osteoarthritis. Nat. Rev. Rheumatol. 2020, 16, 673-688. [CrossRef] 
51. Pfalzer, A.C.; Choi, S.W.; Tammen, S.A.; Park, L.K.; Bottiglieri, T.; Parnell, L.D.; Lamon-Fava, S. S-adenosylmethionine mediates inhibition of inflammatory response and changes in DNA methylation in human macrophages. Physiol. Genom. 2014, 46, 617-623. [CrossRef] [PubMed]

52. Lopez, H.L. Nutritional interventions to prevent and treat osteoarthritis. Part II: Focus on micronutrients and supportive nutraceuticals. PMR 2012, 4, 155-168. [CrossRef] [PubMed]

53. Rutjes, A.W.; Nüesch, E.; Reichenbach, S.; Jüni, P. S-Adenosylmethionine for osteoarthritis of the knee or hip. Cochrane Database Syst. Rev. 2009, CD007321. [CrossRef]

54. De Silva, V.; El-Metwally, A.; Ernst, E.; Lewith, G.; Macfarlane, G.J.; Arthritis Research Campaign working group on complementary and alternative medicines. Evidence for the efficacy of complementary and alternative medicines in the management of fibromyalgia: A systematic review. Rheumatology 2010, 49, 1063-1068. [CrossRef] [PubMed]

55. Li, N.; Zhang, H.H.; Wang, S.H.; Zhu, W.M.; Ren, J.A.; Li, J.S. S-Adenosylmethionine in treatment of cholestasis after total parenteral nutrition: Laboratory investigation and clinical application. Hepatobiliary Pancreat. Dis. Int. 2002, 1, 96-100. [PubMed]

56. Yang, H.P.; Ara, A.I.; Magilnick, N.; Xia, M.; Ramani, K.; Chen, H.; Lee, T.D.; Mato, J.M.; Lu, S.C. Expression pattern, regulation e function of methionine adenosyltranferase 2 beta alternative splicing variants in hepatoma cells. Gastroenterology 2008, 134, 281-291. [CrossRef]

57. Mosca, L.; Pagano, M.; Ilisso, C.P.; Delle Cave, D.; Desiderio, V.; Mele, L.; Caraglia, M.; Cacciapuoti, G.; Porcelli, M. AdoMet triggers apoptosis in head and neck squamous cancer by inducing ER-stress and potentiates cell sensitivity to cisplatin. J. Cell. Physiol. 2019, 234, 13277-13291. [CrossRef]

58. Mosca, L.; Minopoli, M.; Pagano, M.; Vitiello, F.; Carriero, M.V.; Cacciapuoti, G.; Porcelli, M. Effects of S-adenosyl-L-methionine on the invasion and migration of head and neck squamous cancer cells and analysis of the underlying mechanisms. Int. J. Oncol. 2020, 56, 1212-1224. [CrossRef] [PubMed]

59. Pagano, M.; Mosca, L.; Vitiello, F.; Ilisso, C.P.; Coppola, A.; Borzacchiello, L.; Mele, L.; Caruso, F.P.; Ceccarelli, M.; Caraglia, M.; et al. Mi-RNA-888-5p is involved in S-adenosylmethionine antitumor effects in laryngeal squamous cancer cells. Cancers 2020, 12, 3665-3687. [CrossRef]

60. Mosca, L.; Pagano, M.; Vitiello, F.; Ilisso, C.P.; Coppola, A.; Borzacchiello, L.; Cacciapuoti, G.; Porcelli, M. AdoMet synergistically enhances the chemotherapeutic action of cisplatin and improves drug efficacy in JHU-SCC-011 laryngeal squamous cell carcinoma. Nov. Appro. Can. Study 2020, 5, 445-449.

61. Zhou, Y.Y.; Li, Y.; Jiang, W.Q.; Zhou, L.F. MAPK/JNK signaling: A potential autophagy regulation pathway. Biosci. Rep. 2015,35, e00199. [CrossRef]

62. Coppola, A.; Ilisso, C.P.; Stellavato, A.; Schiraldi, C.; Caraglia, M.; Mosca, L.; Cacciapuoti, G.; Porcelli, M. S-Adenosylmethionine inhibits cell growth and migration of triple negative breast cancer cells through upregulating MiRNA-34c and MiRNA-449a. Int. J. Mol. Sci. 2021, 22, 286-304. [CrossRef]

63. Mosca, L.; Vitiello, F.; Borzacchiello, L.; Coppola, A.; Tranchese, R.V.; Pagano, M.; Caraglia, M.; Cacciapuoti, G.; Porcelli, M. Mutual correlation between Non-Coding RNA and S-Adenosylmethionine in human cancer: Roles and therapeutic opportunities. Cancers 2021, 13, 3264. [CrossRef]

64. González-González, R.; Ortiz-Sarabia, G.; Molina-Frechero, N.; Salas-Pacheco, J.M.; Salas-Pacheco, S.M.; Lavalle-Carrasco, J.; López-Verdín, S.; Tremillo-Maldonado, O.; Bologna-Molina, R. Epithelial-mesenchymal transition associated with head and neck squamous cell carcinomas: A review. Cancers 2021, 13, 3027. [CrossRef] [PubMed]

65. Mosca, L.; Pagano, M.; Borzacchiello, L.; Mele, L.; Russo, A.; Russo, G.; Cacciapuoti, G.; Porcelli, M. S-Adenosylmethionine increases the sensitivity of human colorectal cancer cells to 5-fluorouracil by inhibiting P-glycoprotein expression and NF- $\mathrm{B}$ activation. Int. J. Mol. Sci. 2021, 22, 9286. [CrossRef] [PubMed]

66. Sha, J.; Bai, Y.; Ngo, H.X.; Okui, T.; Kanno, T. Overview of evidence-based chemotherapy for oral cancer: Focus on drug resistance related to the epithelial-mesenchymal transition. Biomolecules 2021, 11, 893. [CrossRef] [PubMed]

67. Kabzinski, J.; Maczynska, M.; Majsterek, I. MicroRNA as a novel biomarker in the diagnosis of head and neck cancer. Biomolecules 2021, 11, 844. [CrossRef] 